\title{
A Confidence Measure for Boundary Detection and Object Selection
}

William A. Barrett

william_barrett@byu.edu

Eric N. Mortensen

Follow this and additional works at: https://scholarsarchive.byu.edu/facpub

Part of the Computer Sciences Commons

\section{Original Publication Citation}

E.N. Mortenson, W.A. Barrett "A Confidence Measure for Boundary Detection and Object Selection," Computer Vision and Pattern Recognition 21, Vol. 1, P. 477-484, December, 21.

\section{BYU ScholarsArchive Citation}

Barrett, William A. and Mortensen, Eric N., "A Confidence Measure for Boundary Detection and Object Selection" (2001). Faculty Publications. 555.

https://scholarsarchive.byu.edu/facpub/555 


\title{
A Confidence Measure for Boundary Detection and Object Selection
}

\author{
Eric N. Mortensen ${ }^{1,2}$ (enm@cs.orst.edu) William A. Barrett ${ }^{1}$ (barrett@cs.byu.edu) \\ ${ }^{1}$ Brigham Young University \\ ${ }^{2}$ Oregon State University
}

\begin{abstract}
We introduce a confidence measure that estimates the assurance that a graph arc (or edge) corresponds to an object boundary in an image. A weighted, planar graph is imposed onto the watershed lines of a gradient magnitude image and the confidence measure is a function of the cost of fixed-length paths emanating from and extending to each end of a graph arc. The confidence measure is applied to automate the detection of object boundaries and thereby reduces (often greatly) the time and effort required for object boundary definition within a userguided image segmentation environment.
\end{abstract}

\section{Introduction}

Detection of object boundaries and the subsequent segmentation of objects in digital images are fundamental issues for many computer vision and image analysis techniques. The immense volume of literature that has focused on edge detection and segmentation is one indicator of their essential nature to many image understanding applications and attests to the fact that even incremental improvements to edge detection and segmentation can have far reaching effects.

Despite the large amount of research, no fully automatic technique currently exists that correctly identifies objects of interest in a general class of images. In fact, it is likely that, for certain task-specific applications, general image segmentation will continue to require some amount of human intervention for the next several years at least. As such, one objective of computer vision research should be to reduce the human effort required for accurate and reliable object definition by exploiting high-level visual expertise while requiring minimal user interaction.

The goal of edge detectors-such as those by Hueckel [9], Marr-Hildreth [10], Canny [3], Nalwa-Binford [15], Elder-Zucker [5], Ruzon-Tomasi [17], and others (see $[1,8,19]$ for surveys/comparisons of various edge detectors) -is to find transitions in an image's intensity/color that correspond to meaningful image features. Such techniques typically categorize each pixel's "edgeness" by applying a discontinuity measure-such as the derivative of Gaussian [3,5], parametric model matching [9,15], or compass operator [17]. Since the response of these discontinuity measures to an edge is typically spread across some finite area due to the imaging system's point spread and/or the operator extent and/or intentional pre-smoothing of the image, such detectors often localize edges by either (1) identifying the crestlines of the discontinuity image (via directed non-maximal suppression) $[3,17]$, (2) finding the zero-crossings of the $2^{\text {nd }}$ derivative (or Laplacian) of Gaussian image [10,5], or (3) direct parametric estimation of the edge position $[9,15]$. Finally, many edge detectors then apply a binary decision function to the localized discontinuity response to create a binary edge image.

In the continuous domain, finding the crestlines of a discontinuity image is almost equivalent ${ }^{1}$ to computing the watershed lines that partition the image into catchment basins $[2,14,18]$. In real-world images, the standard watershed transform generally over-segments an image into a collection of small regions, especially in areas where a low SNR produces a plethora of local minima in the gradient magnitude image. However, since watershed lines generally correspond well with meaningful image features, the resulting partition allows image analysis techniques to raise the granularity of the image primitive above the individual pixel (whose spatial arrangement and limited characteristics are controlled by the imaging device) and thereby reformulate their solution in terms of object-centered regions-where the regions' shape, arrangement, and color attributes are determined by the image's underlying scene properties.

Given a weighted graph that is imposed onto an image's gradient watershed, this paper presents a measure that estimates the confidence that a graph arc corresponds to an object or image feature boundary. The arc confidence exploits the watershed's object-centered segmentation to create a measure that utilizes both region- and boundarybased features as well as an internal curvature feature. The resulting measure facilitates two extensions to tobogganbased Intelligent Scissors [13] that reduce (often greatly) both the positioning time and number of seed points required for accurate object boundary definition. An object snapping technique, called confidence snapping, allows for faster seed point placement by snapping the free-point to the nearest position on a high confidence graph arc while a path extension technique extends a partial boundary contour along a high confidence graph path to automatically complete a closed boundary around arbitrarily large objects.

1. The difference is that each end of a watershed line is connected, thereby separating two distinct regions, whereas the crestlines created by directed non-maximal suppression [3] will often dangle (at least one end of the crestline is not connected). One reason is that the watershed transform will extend low elevation crestlines up the side of higher terrain features to connect it with a higher crestline. Thus, hysteresis thresholding of the watershed lines will often produce "barbs" down these crestline extensions [14] while directed non-maximal suppression [3] will typically not identify these extensions as potential edges. 


\section{Graph Arc Confidence}

As mentioned, both confidence snapping and path extension rely on an arc confidence measure that can identify and extend high confidence arcs. The arc confidence measure, in turn, relies on another measure called the branch extension cost, which computes the minimum cumulative cost path from among all possible fixed length paths extending out from an arc. However, before defining the branch extension cost and arc confidence, a brief description of the weighted graph and associated path computations is helpful.

\subsection{Graph Formulation}

Like [13], we impose a weighted planar graph onto the crestlines (i.e., watershed lines) of a gradient magnitude image, $G$. The watershed of $G$ is computed via a 4-connected implementation of tobogganing [6] which results in 4-connected regions where each pixel is labeled according to its catchment basin. The resulting watershed lines, and subsequently the imposed graph, fall on the interpixel grid. Thus, graph vertices, $v$, occur where three or four distinct regions meet at a pixel corner and bidirectional weighted arcs, $\boldsymbol{a}=\left(v_{\text {beg }}, v_{\text {end }}, l_{l}, l_{r}\right)$, are created along the pixel cracks corresponding to the watershed line separating two adjacent catchment basins such that, when traversing the arc from $v_{\text {beg }}$ to $v_{\text {end }}$, the pixels on the left are labeled $l_{l}$ while the pixels on the right belong to region $l_{r}{ }^{1}$.

While the crestline corresponding to an arc is crack based, an arc's weight is pixel based. Let the tuple $(\boldsymbol{p}, \boldsymbol{q})$ define the crack between two adjacent pixels, $p$ and $q$, and let $a_{\text {crac: }}=\left(\left(p_{1}, q_{1}\right),\left(p_{2}, q_{2}\right), \ldots,\left(p_{n}, q_{n}\right)\right)$ be the ordered chain of cracks that traces the crestline corresponding to an arc such that $\operatorname{label}\left(\boldsymbol{p}_{i}\right)=l_{l}$ and $\operatorname{label}\left(\boldsymbol{q}_{i}\right)=l_{r}$ for $1 \leq i \leq n$, then an arc's path vector is the non-repeating 8connected sequence of pixels $a_{\text {path }}=\left(r_{1}, r_{2}, \ldots, r_{m}\right)$ where $m \leq n$ and

$$
\boldsymbol{r}_{j}=\left\{\begin{array}{l}
\boldsymbol{p}_{i} ; \text { if } G\left(\boldsymbol{p}_{i}\right)>G\left(\boldsymbol{q}_{i}\right) \\
\boldsymbol{q}_{i} ; \text { otherwise }
\end{array} .\right.
$$

such that $j \leq \mathrm{i}, \boldsymbol{r}_{j+1} \neq \boldsymbol{r}_{j}$ for $1 \leq j \leq m-1$.

Since a path can traverse an arc in either direction, the pixels in an arc's path vector need to be indexed in either direction. Thus, the $i^{\text {th }}$ directional pixel in an arc's path vector is given by

$$
r_{i}(a, d)= \begin{cases}a_{p a t h}(i) ; & \text { if } d=\text { True } \\ a_{p a t h}(m-i+1) ; & \text { otherwise }\end{cases}
$$

where $d$ is the traversal direction-True for forward (i.e., from $v_{b e g}$, to $\left.v_{\text {end }}\right)$ and False for backward. Further, the length of a path is equal to the number of pixels in all the arcs included in the path. If $C$ is the fixed path length and the path already has $C-m^{*}$ pixels, then $m^{\prime}=\min \left(m, m^{*}\right)$ is the number of pixels still needed to compute the fixedlength path cost.

1. The Iabels are necessary in the arc definition since it is possible to have more that one arc between two adjacent vertices.
Finally, for notational convenience, let

$$
v_{a}^{+}(d)= \begin{cases}v_{e n d} ; & \text { if } d=\text { True (Forward) } \\ v_{b e g} ; & \text { otherwise }\end{cases}
$$

be $a$ 's "forward" vertex relative to the direction specified and let

$$
d_{a}(v)= \begin{cases}\text { True } ; & \text { if } v=v_{b e g} \\ \text { False; } & \text { otherwise }\end{cases}
$$

indicate the traversal direction of $\boldsymbol{a}$ relative to one of its vertices.

\subsection{Arc Weight/Cost}

The cost (or partial cost) for an arc $\boldsymbol{a}$ is a summation of three cost functionals and is given by

$f\left(\boldsymbol{a}, d, \boldsymbol{Q}, m^{\prime}\right)=\sum_{i=1}^{m^{\prime}}\left[f_{g_{i} i}(\boldsymbol{a}, d)+f_{t}(\boldsymbol{a})+f_{b, i}(\boldsymbol{a}, d, \boldsymbol{Q})\right](5)$

where $f_{g, i}, f_{t}$, and $f_{b, i}$ are the gradient magnitude, student's $t$-test, and bending cost functionals, respectively, and $Q$ is a circular queue of the previous 8 points in the path.

The gradient magnitude cost functional is

$$
f_{g, i}(\boldsymbol{a}, d)=\omega_{g}\left[1-\frac{G\left(r_{i}(\boldsymbol{a}, d)\right)}{\max (G)}\right]
$$

where $\omega_{g}$ is the component weight and $G$ is the gradient magnitude of the image - as computed from a multi-scale derivative of Gaussian operator.

The student's $t$-test [16] is a region-based cost functional given by

$$
f_{t}(a)=\omega_{t} \cdot p\left(\mu_{l}=\mu_{r}\right)
$$

where $\omega_{t}$ is the component weight and $p\left(\mu_{l}=\mu_{r}\right)$ is the probability that the distribution of pixel colors in the two watershed regions on either side of the arc (i.e., with labels $l_{l}$ and $l_{r}$ ) have the same mean. The null hypothesis, $H_{0}$, is that the underlying unimodal Gaussian distributions have the same mean (i.e., $H_{0}: \mu_{1}=\mu_{r}$ ); thus, a low probability indicates that the samples are from different distributions and should therefore produce a low cost.

Unlike the external (i.e., data dependent) cost functionals $f_{g, i}$ and $f_{t}$, the bending cost, $f_{b, i}$, is an internal measure that favors low curvature paths. The bending cost is

$$
f_{b, i}(a, d, \boldsymbol{Q})=\frac{\omega_{b}}{\pi} \arccos \left|\frac{\left(r_{i}(\boldsymbol{a}, d)-\boldsymbol{Q}_{4}\right) \cdot\left(\boldsymbol{Q}_{4}-\boldsymbol{Q}_{8}\right)}{\left\|r_{i}(\boldsymbol{a}, d)-\boldsymbol{Q}_{4}\right\|\left\|\boldsymbol{Q}_{4}-\boldsymbol{Q}_{8}\right\|}\right|
$$

where the circular point queue, $Q$, is used to compute changes in path direction by measuring the angular difference between two 4-pixel-long line segments: the first one is between the current pixel and 4 pixels back in the path and the second line segment is between 4 and 8 pixels back in the path vector ${ }^{2}$. The point queue is updated with the current pixel after each computation of $f_{b, i}$.

2. For computational and memory efficiency, $Q$ is implemented as a 24 bit chain code ( 3 bits/pixel) and ( 8 ) is computed using table lookups on each 12 bit half of the chain code. 


\subsection{Branch Extension Cost}

Three quantities are used to compute an arc's confidence: the average directional local cost, the branch extension cost for each branch of the arc, and the path that minimizes the branch extension cost. Given an arc $\boldsymbol{a}$ and a traversal direction $d, a$ 's average directional arc cost is

$$
\overline{f(a, d)}=\frac{1}{m} f\left(a, d, Q_{i n i r}(a, d), m\right)
$$

where $\boldsymbol{Q}_{\text {init }}$ is initialized to minimize $f_{b, i}$ for the first step in the cost computation.

The branch extension cost, $\xi_{(i)}$, explores all possible fixed-length paths extending out through a specific branch of an arc. For a "source" arc $a$ and a traversal direction $d$, $\xi_{(i)}$ first computes $\overline{f(a, d)}$ - which ensures that the point queue is initialized properly-and then invokes a recursive function for each arc (except the source arc) emanating from the forward vertex, $v_{a}^{+}(d)$, of the source arc. That is to say, let $Q^{\prime}$ be the point queue resulting from (9) and let $\operatorname{arc}(v)$ be the set of arcs connected to vertex $v$, then the branch extension cost for each $\operatorname{arc} \boldsymbol{a}_{i}^{\prime} \in \operatorname{arc}\left(v_{a}^{+}(d)\right)-\{\boldsymbol{a}\}$ is

$$
\xi_{(i)}(a, d)=\frac{1}{C} \xi\left(a^{\prime}{ }_{i}, d, Q^{\prime}, C\right)
$$

where $C$ is the fixed (constant) path length and

$$
\begin{aligned}
& \xi\left(\boldsymbol{a}, d, \boldsymbol{Q}, m^{*}\right)=f\left(\boldsymbol{a}, d, \boldsymbol{Q}, \boldsymbol{m}^{\prime}\right)+ \\
& \min _{\boldsymbol{a}_{i}^{\prime}}\left[\xi\left(\boldsymbol{a}_{i}^{\prime}, d_{a}\left(v_{a}^{+}(d)\right), \boldsymbol{Q}^{\prime}, m^{*}-m^{\prime}\right)\right]
\end{aligned}
$$

is the recursive extension cost, which searches all paths of length $C$ that extend out from one end of the arc (as specified by the direction $d$ ) to compute the minimum cumulative cost branch path. Recursion terminates when $m^{*}=0$.

Note that the parameter $\boldsymbol{a}_{i}{ }_{i}$ on the right hand side (RHS) of $(10)$ corresponds to a first level branch of the source arc; it is therefore not equal to the $\boldsymbol{a}_{i}^{\prime}$ in the minimum term of (11), which correspond to second, third, etc., level branches. Also, the $Q^{\prime}$ in the minimum term of $(11)$ is the point queue resulting from the local cost computation, $f(a, d, Q, m)$, and becomes the $Q$ parameter in the next level of recursion. Since the computational complexity of (11) grows exponentially with $C$, it is a good idea to keep this constant fairly small. (By default, $C=8$.)

Finally, let $\xi_{\min }$ be the branch extension cost for the branch that minimizes $\xi_{(i)}$ and let

$$
\rho_{(i)}(a, d)=\left(a_{0}, a_{1}, a_{2}, \ldots, a_{n}\right)
$$

be the path that minimizes $\xi_{(i)}(a, d, Q)$, where $a_{0}=a$ (the source arc) and $a_{1}=a_{i}^{\prime}$ (the first branch arc as given in the RHS of equation (10)).

\subsection{Arc Confidence}

Given two adjacent arcs, $\boldsymbol{a}$ and $\boldsymbol{a}^{\prime}$, that emanate from a vertex $v$, let $d^{\prime}=d_{\boldsymbol{a}}(v)$ and let $\boldsymbol{a}^{\prime}$ correspond to the branch index $i$ relative to $a$ such that $\xi_{(i)}\left(a, d^{\prime}\right)$ is the minimum cost for the branch $\boldsymbol{a}^{\prime}$, then

$$
\operatorname{conf}_{\text {diff }}\left(a, a^{\prime}\right)=\left[1-\frac{\xi_{(i)}\left(a, d^{\prime}\right)-\xi_{\min }\left(a, d^{\prime}\right)}{1-\xi_{\min }\left(a, d^{\prime}\right)}\right]^{2}
$$

is the differential confidence, which measures the confidence that branch $\boldsymbol{a}^{\prime}$ is the lowest cost extension of $\operatorname{arc} \boldsymbol{a}$. The numerator in the RHS of (13) measures the cost difference between the specified branch and the minimum cost branch - thus the minimum cost branch will always return a maximum confidence. The denominator scales the cost difference such that a branch with the largest absolute cost will still have maximal differential cost while a minimum cost branch will have zero cost difference (thus maximizing the differential cost's discriminatory capacity). Since a low cost difference should produce a high confidence, the scaled differential cost is flipped by subtracting it from 1. Finally, the differential confidence is squared to penalize costs that are close to maximum.

The composite branch confidence is given by

$$
\operatorname{con} f_{(i)}(a, d)=w_{(i)}(a, d) \prod_{j=1}^{n} \operatorname{con} f_{d i f f}\left(\rho_{(i), j}, \rho_{(i), j-1}\right)(14)
$$

where

$$
w_{(i)}(\boldsymbol{a}, d)=1-\xi_{(i)}^{2}(\boldsymbol{a}, d)
$$

and combines the flipped branch extension cost with the product of the backward differential confidence for each arc in the path of the minimum cost branch extension. The use of the product ensures that the confidence measure does not exceed unity. While (12) gives the path corresponding to the minimum cost branch extension cost from $a$ through $a_{i}^{\prime}=a_{1}$ to $a_{n}$, it is not necessarily the case that the reverse path produces the minimum cost branch extension from $a_{n}$ back to $a$. Thus, the product in the above equation measures the combined confidence that the reverse path back to $a$ represents the minimum (or near minimum) cost branch extension of $a_{n}$. The product is weighted by the compliment of the branch extension cost where the square favors lower cost extensions.

By enforcing a bidirectional consistency along the path that minimizes $(10)$, $\operatorname{conf}_{(i)}$ is the primary mechanism for reducing false positives - such as the "barbs" produced by applying hysteresis thresholding to the watershed lines [14]. As such, (14) is central in the confidence measure.

Finally, the overall arc confidence is given by

$$
\operatorname{conf}(\boldsymbol{a}) \underset{d \in\{\text { False, True }\}}{ }\left[w(\boldsymbol{a}, d) \cdot \max _{i}\left(\operatorname{conf} f_{(i)}(\boldsymbol{a}, d)\right)\right],
$$

which computes the product, over both arc traversal directions, of the weighted maximum composite branch confidence for the given traversal direction. The weight for each direction is simply

$$
w(\boldsymbol{a}, d)=1-\overline{f(\boldsymbol{a}, d)}
$$

where $\overline{f(a, d)}$ is the average arc cost given in (9).

\section{Object Selection via Path Extension}

We use the arc confidence measure to automate the detection of object boundaries and thereby reduce (often greatly) the human input required for object boundary definition using Intelligent Scissors [11-13]. Based on livewire optimal path selection, Intelligent Scissors allows a 
user to rapidly and accurately define piece-wise globally optimal contour segments that correspond to a desired object boundary. Given a weighted graph representation of an image and a user-specified "seed" vertex, the live-wire interactively displays the optimal (minimum cost) path from the current cursor position (or free-point) back to the seed point. The live-wire uses an efficient, $O(N)$ implementation of Dijkstra's algorithm [4] to compute, in real time, the minimum-path spanning tree rooted at a seed vertex.

Since a free-point specifies only one path back to a seed point, defining a closed contour with Intelligent Scissors traditionally requires at least three user-specified pointsand generally only if the object boundary is simple and (mostly) well defined. Normally, the three point minimum consists of two seed points and one free-point where the closed boundary consists of the path connecting the two seed points (created previously via live-wire) and the two paths connecting the free-point with each seed. However, when the free-point is positioned on a non-spanning graph arc (i.e., an arc that is not included in the minimum path spanning tree, see Fig. 1), [13] can satisfy the three point minimum by "splitting" the free-point into two virtual free-points, one at each vertex connected by the arc.

While it is possible to define an object boundary in this manner, it typically requires precise cursor positioning to select the desired non-spanning edge (which may be only one or two pixel cracks long). Further, this technique requires that the object boundary contain only one nonspanning graph arc-i.e., of the graph arc's defining an object boundary, all but one are included in a seed point's minimum-path spanning tree. Thus, the free-point splitting used in [13] has no possibility of defining many object boundaries, such as the ' $U$ ' in Fig. 1, with just one seed since they contain more than one non-spanning arc regardless of the seed point placement.

\subsection{Live-Wire Path Extension}

The same effect can be achieved while allowing the user much more freedom in positioning the free-point by using the arc confidence measure to extend the current live-wire path past the free-point to create a virtual free-point several tens, or even hundreds, of pixels away from the current free-point. Given a free-point vertex and the optimal path map for the most recent seed, live-wire path extension uses the optimal path from the free-point to determine the beginning arc and traversal direction, which is in the opposite direction of the optimal path. It then iteratively extends the live-wire path by finding the next (adjacent) arc that maximizes the composite branch confidence measure given in (14). If the next arc's confidence is above a threshold value, it adds the arc to the ordered sequence that defines the extension and continues extending the path from this new arc. Path extension continues, often for tens to hundreds of pixels, until reaching a point where it can define a closed, non-overlapping boundary or until the arc confidence falls below a minimum threshold.

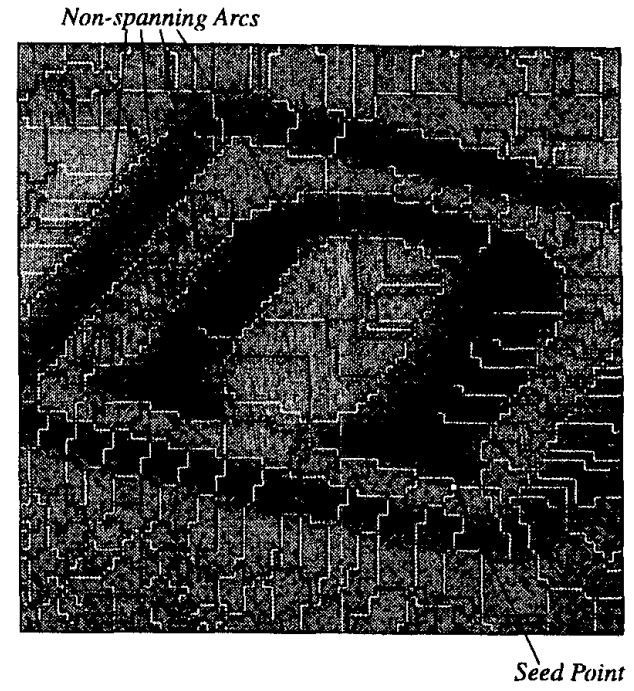

Figure 1: Object boundary with multiple non-spanning arcs. Arcs included in the minimum-path spanning tree are white while non-spanning arcs are black. Regardless of where the seed point is placed, the boundary of the ' $U$ ' will contain more than one nonspanning arc.

The minimum acceptable confidence threshold is a monotonically increasing function of the estimated standard deviation of the image noise ${ }^{l}, s_{n}$, and is given by

$$
c_{\min }=\frac{1}{2}\left(1-\frac{b_{\min }}{b_{\min }+s_{n}}\right)
$$

where $b_{\min }$ controls the steepness of the curve. (18) is formulated to give a low value for images that have very little noise while $c_{\min }$ approaches $1 / 2$ as the noise increases.

\subsection{Virtual Free-Point Placement}

The purpose of live-wire path extension is to create a second, virtual free-point (hereafter called the virtualpoint) at the far end of the extension. The optimal path segments from the two free-points and the path extension between them creates a closed boundary, allowing many object boundaries to be defined with only a single seed. To create a closed, non-overlapping contour that (ideally) defines an object boundary, path extension continues until the optimal path from the virtual-point does not overlap with the free-point's live-wire path and as long as it does not overlap with itself or previously defined segments.

To ensure that the virtual-point's optimal path does not include any of the arcs from the free-point's optimal path, live-wire path extension must continue until encountering an arc that belongs to a different major branch of the minimum-path spanning tree than the major branch containing the free-point. A minimum-path spanning tree is composed of 2 to 4 major branches depending on how many arcs emanate out from (i.e., are adjacent to) the seed ver-

1. $s_{n}$ is estimated automatically by fitting a Gaussian distribution to a portion of a smoothed, pixel-difference histogram. 
tex. Arcs that are adjacent to the seed point are primary arcs. Each major branch consists of a primary arc and all the arcs and vertices that are descendents (with respect to the tree structure) of the primary arc. Thus, the optimal path from a point on a major branch must include the primary arc for that branch and consequently, the optimal paths from any two points on the same major branch must, at some point, overlap (and continue to overlap until reaching the seed point). To quickly determine which major branch any given graph vertex is on, the branch index of each primary arc is propagated to every vertex during creation of the minimum-path spanning tree. Thus, each vertex knows which major branch it belongs to, thereby allowing immediate comparison.

For example, Figure 2(a) illustrates a case where the minimum-path spanning tree for an order-three seed vertex (on the boundary of the ' $U$ ') produces three major branches and each spanning arc and vertex is shaded either black, white, or light gray according to its major branch. Thus, given the free-point position shown in Fig. 2(b), live-wire path extension (which starts in the "black" branch) follows the boundary of the 'U' (Fig. 2(b-d)) until subsuming the non-spanning arc (Fig. 2(d)) that causes it to jump to a new, unused major branch (i.e., the white branch). Since the vertex after the fourth non-spanning arc is on a different major branch, the branching criterion is now satisfied and the algorithm terminates, positioning the virtual-point after the non-spanning arc (Fig. 2(f)), which, with the addition of the optimal path from the virtualpoint, closes the contour defining the object boundary.

In order to prevent live-wire path extension from overlapping with itself or other contours segments (including the current live-wire and any previously defined boundary segments), each vertex included in the current live-wire path as well as those in the path extension and any previously defined contour segments is marked. Path extension terminates upon encountering a marked vertex.

Finally, Figure 3 shows the results obtained with livewire path extension on several objects. Each boundary is defined with a single seed point and, in most cases, the free-point has moved just a few $(<10)$ pixels away from the seed point (just enough to specify a live-wire path). As can be seen, live-wire path extension is able to automatically define large segments of the object boundary. In fact, if the branch termination criteria is turned off, path extension will automatically circumnavigate the majority of the object boundaries in Fig. 3. Consequently, when combined with confidence snapping (Section 4), these boundaries can be defined many times faster (on the order of 4 to $20+$ times) and with much less user interaction than is required without the advantage of the confidence measure.

\section{Confidence Threshold Snapping}

Traditionally, seed point placement has been one of the more time consuming and tedious aspects of object selection with Intelligent Scissors. To alleviate seed point placement, the original Intelligent Scissors $[11,12]$ snaps the free-point to the "strongest" (maximum gradient mag-
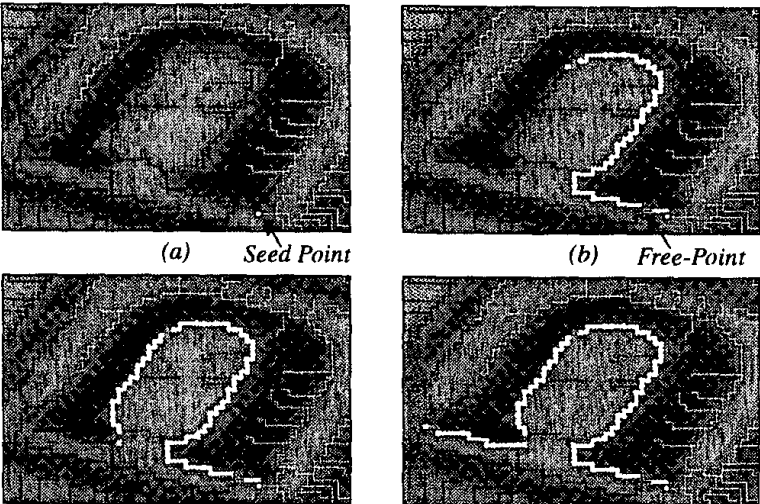

(b) Free-Point (c)

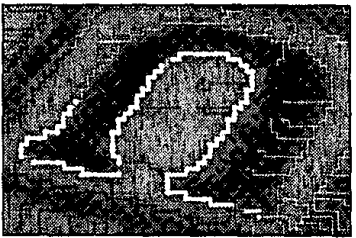

(e)

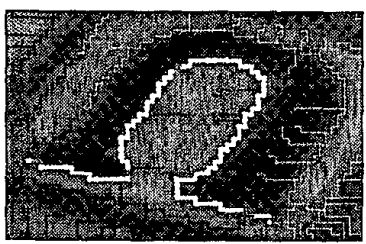

(d)

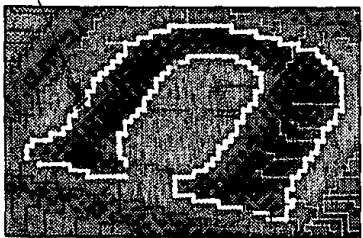

$(f)$
Figure 2: Example live-wire path extension. (a) The minimum-path spanning tree resulting from a seed point placed on the "U". Black, white, and light gray arcs are colored according to their major branch while non-spanning arcs are not shown. Notice that the " $U$ " boundary contains four gaps corresponding to non-spanning arcs (as identified in Fig. 1). (b) The free-point is positioned on the "U" just slightly left of the seed point (in the black major branch) and the live-wire between the free-point and the seed point extends (thick white) to include the first non-spanning arc (thick light gray). Since the non-spanning arc does not jump to a new major branch, path extension continues on to the (c) second and (d) third non-spanning arcs before arriving at the (e) fourth non-spanning arc, which does jump to an unused major branch (i.e., the white branch). (f) The virtual-point is placed after the fourth non-spanning arc and the optimal path from the virtual-point closes the object boundary.

nitude) pixel within an interactively adjustable square region (up to 9 pixels away). A similar snapping policy is implemented in [7] using multi-scale gradient descent.

However, snapping to the strongest point in a neighborhood creates snap points that dominate a neighborhood, often at the expense of "good" snap points that are closer to the mouse pointer. Ideally, cursor snapping should position the free-point on the closest point that is deemed to be "good enough" (i.e., it is considered to be on an object boundary).

In addition to providing a means for extending the livewire path, the arc confidence provides a measure for determining if an are is "good enough" for cursor snapping. The basic idea is to search for all the arcs within the specified neighborhood and snap to the closest point on an arc whose confidence exceeds a threshold value. Consequently, this technique is called confidence threshold snapping (CTS). 


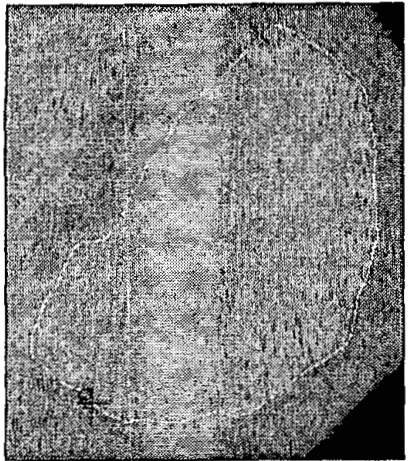

(a)

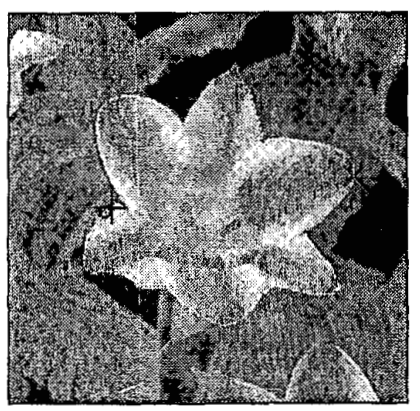

(d)

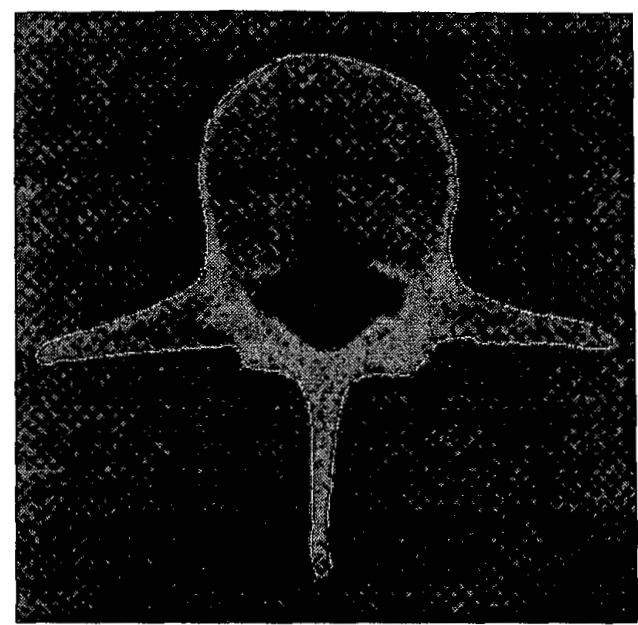

(b)

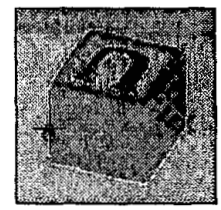

(e)

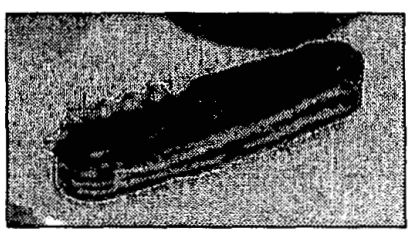

$(f)$

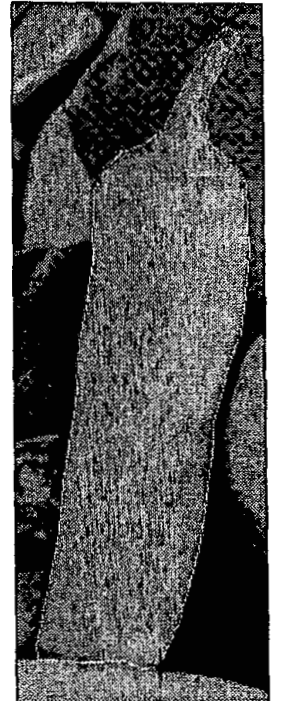

(c)

Figure 3: Boundary definition results using live-wire path extension. Each boundary is defined in a fraction of a second with only one seed point (black circle). In each case, the free-point (black cross-hair) is positioned (via confidence snapping, Section 4) on the object boundary only a short distance from the seed point while live-wire path extension automatically defines a large portion of the object boundary and places the virtual-point (black $X$ ) to create a closed object boundary with very little user input. (a) Left ventricle. (b) Spinal vertebrae. (c) Pepper. (d) Tulip. (e) Block and 'U'. (f) Pocket knife.

\subsection{Neighborhood Membership}

The first challenge for CTS is to determine which arcs to include in the neighborhood. Unlike the square neighborhood in pixel-based snapping where neighborhood membership is easily determined, the irregular region structure resulting from tobogganing requires a more sophisticated means of ascertaining neighborhood membership. Also, rather than a square neighborhood, CTS uses a circular neighborhood with a maximum radius of 36 pixels.

Membership is tested for each arc bounding each region during a breadth-first expansion of the region beneath the mouse pointer. Given a mouse position, $\boldsymbol{p}_{m}$, a maximum snap distance, $d_{\max }$, and the label of the region containing the mouse cursor, $l_{m}$. CTS first initializes a FIFO queue with $l_{m}$ and marks the region as being queued. While the queue is not empty, CTS iteratively pulls a region off the front of the queue and checks each arc defining the region boundary for neighborhood membership. An arc belongs in the neighborbood if it has at least one inter-crack point (i.e., a pixel corner on the arc crack list) that is within $d_{\max }$ of $p_{m}$. That is to say, if the minimum distance from a point $p$ to an $\operatorname{arc} e$ is given by

$$
d(a, p)=\min _{i}\|p n t(a, i)-p\|
$$

where pnt $(e, i)$ is the $i^{\text {th }}$ pixel corner in $a$ 's crack list, then $\boldsymbol{a}$ belongs to the neighborhood of $\boldsymbol{p}_{m}$ if $d\left(\boldsymbol{e}, \boldsymbol{p}_{m}\right) \leq d_{\max }$. Since an arc separates two adjacent regions, it is marked after its first encounter to avoid repeated processing. Thus, an arc is ignored if it's outside the neighborhood or already marked.

An arc is processed by computing its confidence and comparing it to the threshold $c_{\max }$. The confidence threshold is given by

$$
c_{\text {max }}=\left(u_{\max }-l_{\max }\right)\left(1-\frac{b_{\max }}{b_{\max }+s_{n}}\right)+l_{\max }
$$

where $u_{\max }$ and $l_{\max }$ specify the upper and lower bounds, respectively, and, like (18), $b_{\max }$ controls the steepness of the curve ${ }^{1}$ and $s_{n}$ is a estimate of the image noise. If an arc's confidence is greater than or equal to $c_{\text {max }}$, the arc is expanded in both directions to create a subtree (Section 4.2). The nearest snap point on the subtree is computed while building the subtree and any arc included in the subtree is marked as processed, producing a barrier that blocks further region expansion. Since there may be multiple subtrees created within a neighborhood, the minimum distance, $d^{\prime}$, and snap point-indicated by an arc, $a^{\prime}$, and a

1. By default, $u_{\max }=0.9375, l_{\max }=0.5$, and $b_{\max }=4$. 
crack index, $i d x^{\prime}$-are initialized/updated during subtree creation, thus ensuring a single minimum-distance snap point for all subtrees in the neighborhood.

If, however, the arc confidence does not meet the threshold, it is not considered a candidate for snapping. Rather, it is considered "spurious" and region expansion continues through the arc by determining the region on the other side of the arc. If the neighboring region has not already been marked, it is added to the end of the queue and marked to avoid multiple insertions into the queue. Finally, the arc is marked to prevent reprocessing it when the region that was just added to the queue is expanded.

Once the closest snap point is computed for a subtree, there seems to be no reason to continue region expansion beyond the minimum snap point distance computed thus far. Setting $d_{\text {max }}$ to $d^{\prime}$ after creating a subtree ${ }^{1}$ reduces the maximum snap distance and produces a smaller search neighborhood.

\subsection{Subtree Creation and Nearest Snap Point}

CTS subtree creation extends an arc by iteratively stepping through the forward vertex to the next arc that maximizes the branch confidence until finding an arc whose confidence does not satisfy the minimum confidence threshold. Unlike live-wire path extension, subtree creation checks at each vertex for a possible subtree branch by computing the branch confidence ratio and comparing it to a confidence ratio threshold, $c_{\text {ratio }}$. The branch confidence ratio is given by

$$
\operatorname{con} f_{\text {ratio }}(a, d)= \begin{cases}1.0 ; & \text { if } \operatorname{conf}\left(a_{j}\right) \geq c_{\max } \\ \frac{\operatorname{con} f_{(i)}(a, d)}{\operatorname{con} f_{(j)}(a, d)} & \text { otherwise }\end{cases}
$$

where

$$
i=\underset{i}{\operatorname{argmax}}\left(\operatorname{con} f_{(i)}(a, d)\right)
$$

and

$$
j=\underset{J \neq i}{\operatorname{argmax}}\left(\operatorname{con} f_{(j)}(a, d)\right)
$$

are the arc indices of the highest and second highest branch confidence, respectively, relative to the forward vertex of $a$ as defined by the traversal direction $d$. If the confidence of the arc $a_{j}$ (corresponding to the second highest branch confidence) is greater than the maximum confidence threshold, then the second most confident branch is, in its own right, a high confidence branch, and the resulting ratio is one. Otherwise, the ratio is greater than one. By default, $c_{\text {ratio }}=2$ indicating that no branching occurs if the highest branch confidence is more than twice that of the next highest, in which case the arc is extended as a single path. However, if the ratio is less than or equal to the threshold then multiple branches are created by recursively calling the subtree function for each forward arc whose confidence is greater than $c_{l o w}$ which is the average

1. In this case the snap radius is set to $d^{\prime}+1$ to create a small buffer zone and to prevent the circle (showing the trimmed snap distance) from interfering with the visualization of the seed arc and snap point of the minimum confidence threshold, $c_{\min }$, and the maximum forward-arc confidence, $\operatorname{conf}\left(\boldsymbol{a}_{i}\right)$. Thus, each call of the subtree function creates a single branch of the tree.

As with path extension, each arc is marked to prevent region expansion through the subtree and branch extension terminates when a new arc to be added is beyond the bounds of the snap neighborhood. To prevent infinite loops, each forward vertex is marked and the branch extension terminates if a marked vertex is encountered.

Figure 4 shows some example snaps with their associated trimmed neighborhoods and high confidence subtrees. In each case the initial snap distance is 36 pixels. Note that in most cases the subtree branches correspond to the boundaries of identifiable image features. Also note that when the snap neighborhood contains more and/or complex subtrees, such as the top-right example, the position of the mouse pointer needs to be, in general, more constrained in order to place the snap point on the desired image boundary, while in less complicated regions, the cursor can be farther from the desired arc (as in the bottom-right example ${ }^{2}$ ). Further, since the snap radius is trimmed after completing each subtree, the first subtree is clipped against the original snap neighborhood while the next subtree is bounded by the trimmed radius resulting from the first subtree.

Finally, computation of both CTS and the live-wire path extension with their associated arc confidences is fast enough (even on moderate PCs) to occur in real time-i.e., it's fast enough to not hinder the interactive responsiveness of the live-wire selection tool. Thus, as the mouse cursor is moved, Intelligent Scissors computes the confidence of each arc as it is needed in a breadth-first expansion, extends high confidence arcs to find the closest snap point on a high confidence subtree, snaps the free-point to the snap point, and, if a prior seed point exists, extends the live-wire path in an attempt to define a closed object boundary. All this is computed at interactive rates.

\section{Conclusions}

This paper has presented a confidence measure that estimates the assurance that a graph arc corresponds to an image feature boundary. The arc confidence exploits the watershed's object-centered partition of the image space to create a measure that utilizes both region- and boundarybased features as well as an internal curvature feature. The resulting measure facilitates two extensions to Intelligent Scissors that further automates general-purpose object selection by reducing (often greatly) both the positioning effort and number of seed points required for accurate boundary definition while allowing for greater freedom in cursor movement and positioning.

2. While the bottom-left example in Fig. 4 also specifies an "uncomplicated" neighborhood, the mouse pointer is positioned to illustrate how the subtree extends the seed arc around the spinal process to place the snap point on the opposite side. A similar snap point would result if the cursor is moved several pixels to the right of the spinal process, as long as it is still within the snap distance 

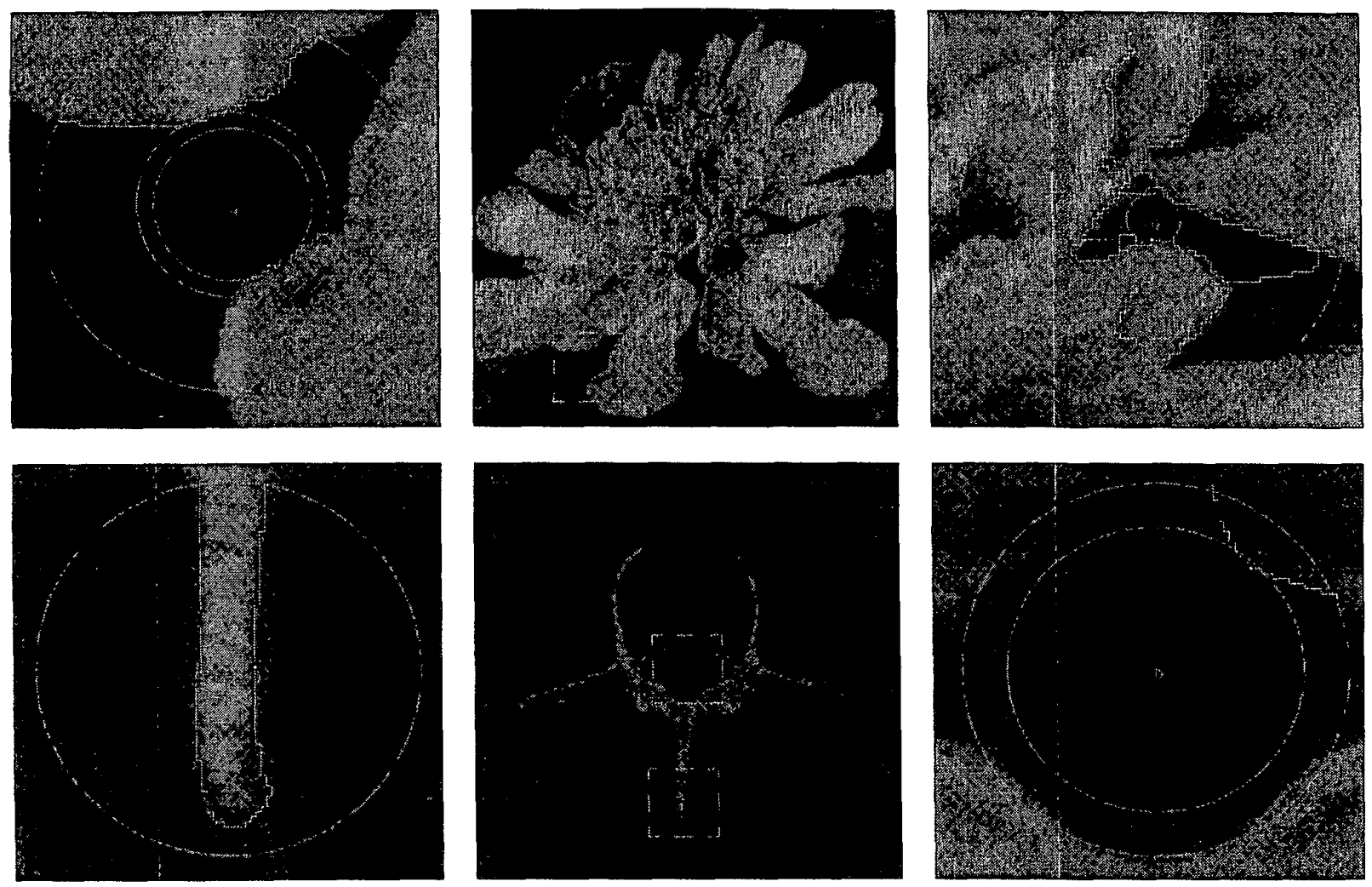

Figure 4: Examples of confidence threshold snapping. The initial snap radius for each example is 36 pixels and the trimmed snap radii are shown as progressively smaller circles. The seed edge for each subtree is shown in red and the snap point is indicated by a whitefilled circle with a red boundary. (Top) Flower. (Bottom) Spinal vertebrae.

\section{References}

11] S. Baker, Design and Evaluation of Feature Detectors, Ph.D. Thesis, Columbia University, 1998

[2] S. Beucher and C. Lantuejoul, "Use of Watersheds in Contour Detection," in Proc. Int'l Workshop Image Processing, Real-Time Edge and Motion Detection/Estimation, 132:2.1-2.12, Sept. 1979.

[3] J. Canny, "A Computational Approach to Edge Detection," IEEE PAMI, 8(6):679-698, Nov. 1986.

[4] E. W. Dijkstra, "A Note on Two Problems in Connexion with Graphs," Numerische Mathematik, 1: 269-270, 1959.

[5] J. H. Elder and S. W. Zucker, "Local Scale Control for Edge Detection and Blur Estimation," IEEE PAMI, 20(7):699-716, July 1998.

[6] J. Fairfield, "Toboggan Contrast Enhancement for Contrast Segmentation," in IEEE ICPR, Vol. 1, pp. 712-716, June 1990.

[7] M. Gleicher, "Image Snapping," in Computer Graphics, pp. 183-190, Aug. 1995

[8] M. Heath, S. Sarkar, T. Sanocki, and K.W. Bowyer, "A Robust Visual Method for Assessing the Relative Performance of EdgeDetection Algorithms" IEEE PAMI, 19(12):1338-1359, Dec. 1997.

[9] M. H. Hueckel, "An Operator Which Locates Edges in Digitized Pictures," Journal of the ACM, 18(1):113-125, Jan. 1971.

[10] D. Marr and E. Hildreth, "Theory of Edge Detection," in Proc. of the Royal Society of London, 207(1167):187-217, Feb. 29, 1980.

[11] E. N. Mortensen and W. A. Barrett, "Intelligent Scissors for Image Composition," in Computer Graphics, pp. 191-198, Aug. 1995.
[12] E. N. Mortensen and W. A. Barrett, "Interactive Segmentation with Intelligent Scissors," GMIP, 60(5): 349-384, Sept. 1998.

[13] E. N. Mortensen and W. A. Barrett, "Toboggan-Based Intelligent Scissors with a Four Parameter Edge Model," in IEEE CVPR, Vol. II, pp. 452-458, June 1999.

[14] L. Najman and M. Schmitt, "Geodesic Saliency of Watershed Contours and Hierarchical Segmentation," IEEE PAMI, 18(12):11631773, Dec. 1996

[15] V. S. Nalwa and T. O Binford, "On Detecting Edges," IEEE PAMI, 8(6):699-714, Nov. 1986

[16] W. H. Press, et al., Numerical Recipes in C (2 ${ }^{\text {nd }}$ ed.), ch. 15, pp. 681-688, Cambridge University Press, 1992.

[17] M. A. Ruzon and C. Tomasi, "Color Edge Detection with the Compass Operator," in IEEE CVPR, Vol. II, pp. 160-166, June 1999.

[18] L. Vincent and P. Soille, "Watersheds in Digital Spaces: An Efficient Algorithm Based on Immersion Simulations," IEEE PAMI, 13(6):583-598, June 1991

[19] D. Ziou and S. Tabbone, "Edge Detection Techniques - An Overview," Int'l. Jour. Pat. Recog. and Image Anal., 8(4):537-559, 1998. 